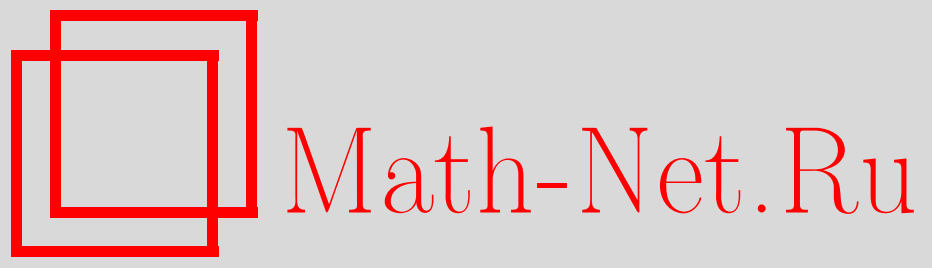

А. А. Боровков, Аппроксимация второго порядка для распределения максимума случайного блуждания с отрицательным сносом и бесконечной дисперсией, Теория вероятн. и ее примен., 2014, том 59, выпуск 1, 5-27

DOI: https://doi.org/10.4213/tvp4548

Использование Общероссийского математического портала Math-Net.Ru подразумевает, что вы прочитали и согласны с пользовательским соглашением http://www . mathnet.ru/rus/agreement

Параметры загрузки:

IP: 54.197 .130 .99

26 апреля 2023 г., 16:18:01

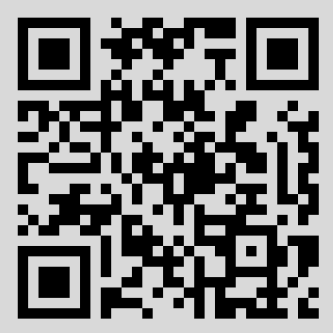




\section{АППРОКСИМАЦИЯ ВТОРОГО ПОРЯДКА ДЛЯ РАСПРЕДЕЛЕНИЯ МАКСИМУМА СЛУЧАЙНОГО БЛУЖДАНИЯ С ОТРИЦАТЕЛЬНЫМ СНОСОМ И БЕСКОНЕЧНОЙ ДИСПЕРСИЕЙ ${ }^{1)}$}

Пусть $\xi, \xi_{1}, \xi_{2}, \ldots$ - независимые одинаково распределенные случайные величины, $a:=-\mathbf{E} \xi>0, S_{n}:=\sum_{j=1}^{n} \xi_{j}, S_{0}=0$, $S:=\sup _{n \geqslant 0} S_{n}, F_{+}(t):=\mathbf{P}(\xi \geqslant t), F_{-}(t):=\mathbf{P}(\xi \leqslant-t), F_{+}^{I}(t)=$ $\int_{t}^{\infty} F_{+}(u) d u$. Хорошо известно (см., например, [1], [5]), что если функция $F_{+}^{I}(t)$ субэкспоненциальна, то $\mathbf{P}(S \geqslant x) \sim F_{+}^{I}(x) / a$ при $x \rightarrow \infty$. В [2] (см. также [3]) при условии $\mathbf{E} \xi^{2}<\infty$ найден следующий член асимптотического разложения для $\mathbf{P}(S \geqslant x)$ при $x \rightarrow \infty$ (порядка $\left.F_{+}(x)\right)$ в случае, когда функция $F_{+}(x)$ либо правильно меняется на бесконечности, либо семиэкспоненциальна. В настоящей работе получена аппроксимация второго порядка для $\mathbf{P}(S \geqslant x)$ в случае, когда $\mathbf{E} \xi^{2}=\infty$, а функции $F_{ \pm}(t)$ удовлетворяют некоторым условиям правильного изменения. Полученные результаты распространены на обобщенные процессы восстановления.

Ключевые слова и фразы: максимум сумм случайных величин; максимум обобщенного процесса восстановления; аппроксимация второго порядка; правильное изменение; асимптотика второго порядка для функции восстановления; большие уклонения сумм случайных величин, не имеющих среднего.

1. Введение и основные результаты. Пусть $\xi, \xi_{1}, \xi_{2}, \ldots$ - независимые одинаково распределенные случайные величины,

$$
\begin{gathered}
a:=-\mathbf{E} \xi>0, \quad S_{n}:=\sum_{j=1}^{n} \xi_{j}, \quad S_{0}=0, \quad S:=\sup _{n \geqslant 0} S_{n}, \\
F_{+}(t):=\mathbf{P}(\xi \geqslant t), \quad F_{-}(t):=\mathbf{P}(\xi \leqslant-t), \quad F_{ \pm}^{I}(t):=\int_{t}^{\infty} F_{ \pm}(u) d u .
\end{gathered}
$$

Хорошо известно (см., например, [1], [5]), что если функция $F_{+}^{I}(t)$ субэкспоненциальна, то

$$
\mathbf{P}(S \geqslant x) \sim \frac{F_{+}^{I}(x)}{a} \quad \text { при } x \rightarrow \infty .
$$

${ }^{*}$ Институт математики им. С. Л. Соболева СО РАН, Новосибирск, Россия; е-mail: borovkov@math.nsc.ru

1) Работа выполнена при поддержке РФФИ (грант № 11-01-00285). 
Обозначим через $\mathscr{R}$ класс правильно меняющихся функций (п.м.ф.) на бесконечности, т.е. класс функций $G(t)$, представимых в виде

$$
G(t)=t^{-\gamma} L(t)
$$

где $L(t)$ - медленно меняющаяся функция (м.м.ф.) при $t \rightarrow \infty$.

В [2], [3, теорема 7.5.3] было установлено следующее утверждение.

Теорема 1.1. Пусть $F_{+} \in \mathscr{R}$ или $F_{+}$семиэкспоненциальна, $\mathbf{E} \xi^{2}<\infty$, распределение $\xi$ нерешетчато. Тогда при $x \rightarrow \infty$

$$
\mathbf{P}(S \geqslant x)=\frac{F_{+}^{I}(x)}{a}+c F_{+}(x)+o\left(F_{+}(x)\right)
$$

где

$$
c=\frac{\mathbf{E} \xi^{2}}{2 a^{2}}-\frac{\mathbf{E} S}{a} .
$$

Соотношение (1.3) сохранится и в решетчатом случае, но при несколько ином значении постоянной $c$ (см. [2], [3, теорема 7.5.3]); более подробную библиографию см. в [3]).

Из представлений (1.3), (1.4) видно, что в случае $\mathbf{E} \xi^{2}=\infty$ асимптотика второго порядка $\mathbf{P}(S \geqslant x)$ (если таковая существует) будет иной, чем в (1.3).

Чтобы сформулировать основное утверждение настоящей работы нам понадобится еще ряд обозначений. Через $\mathscr{R}_{\gamma}$ мы обозначим подкласс п.м.ф. (1.2) с показателем $-\gamma$. Пусть далее

$$
\eta_{+}:=\min \left\{k \geqslant 1: S_{k}>0\right\}, \quad \eta_{-}:=\min \left\{k \geqslant 1: S_{k} \leqslant 0\right\}
$$

$\left(\eta_{ \pm}=\infty\right.$, если соответственно $\left.\max _{k \geqslant 1} S_{k} \leqslant 1, \min _{k \geqslant 1} S_{k}>0\right)$,

$$
\chi_{ \pm}=S_{\eta_{ \pm}}
$$

(случайные величины $\chi_{ \pm}$определены лишь на событиях $\left\{\eta_{ \pm}<\infty\right\}$ ). Положим $\xi_{+}=\max (0, \xi), \xi_{-}=\max (0,-\xi)$, так что $F_{ \pm}(t)=\mathbf{P}\left(\xi_{ \pm} \geqslant t\right)$. Мы будем различать следующие две возможности:

$$
\begin{array}{lll}
{\left[\mathbf{R}_{<\infty}^{\alpha}\right] .} & \mathbf{E} \xi_{-}^{2}<\infty, \quad F_{+} \in \mathscr{R}_{\alpha}, \quad \alpha \in(1,2) ; \\
{\left[\mathbf{R}_{\beta}^{\alpha}\right] .} & \mathbf{E} \xi_{-}^{2}=\infty, \quad F_{-} \in \mathscr{R}_{\beta}, \quad \beta \in(1,2), \quad F_{+} \in \mathscr{R}_{\alpha}, \quad \alpha>1 .
\end{array}
$$

Теорема 1.2. Пусть $a=-\mathbf{E} \xi>0$. Тогда:

(i) если вылолнено условие $\left[\mathbf{R}_{<\infty}^{\alpha}\right], \alpha \in(1,2)$, то при $x \rightarrow \infty$

$$
\mathbf{P}(S \geqslant x)=\frac{F_{+}^{I}(x)}{a}+\frac{2(\alpha-1) I(\alpha)\left(F_{+}^{I}(x)\right)^{2}}{a^{2}}(1+o(1)),
$$

где $I(\alpha):=\int_{0}^{1 / 2} s^{1-\alpha}(1-s)^{-\alpha} d s<\infty$ nри $\alpha \in(1,2)$; 
(ii) если выполнено условие $\left[\mathbf{R}_{\beta}^{\alpha}\right], \alpha \in(1,2), \beta \in(1,2), u F_{-}(x)=$ $O\left(F_{+}(x)\right)$ при $x \rightarrow \infty$, mo

$$
\begin{aligned}
\mathbf{P}(S \geqslant x)= & \frac{F_{+}^{I}(x)}{a}+\frac{2(\alpha-1) I(\alpha)\left(F_{+}^{I}(x)\right)^{2}}{a^{2}}(1+o(1)) \\
& +\frac{C(\alpha, \beta)}{a^{2}} F_{-}^{I}(x) F_{+}^{I}(x)(1+o(1)),
\end{aligned}
$$

где $C(\alpha, \beta):=(\alpha-1) \int_{1}^{\infty} s^{-\alpha}(s-1)^{1-\beta} d s<\infty$ при $\beta \in(1,2)$;

(iii) если выполнень условие $\left[\mathbf{R}_{\beta}^{\alpha}\right], \beta \in(1,2)$, и одно из двух условий:

(b) $\alpha \in(1,2), F_{-}(x) \gg F_{+}(x)$ nрu $x \rightarrow \infty$;

(c) $\alpha>2, \alpha \neq 3$,

mo

$$
\mathbf{P}(S \geqslant x)=\frac{F_{+}^{I}(x)}{a}+\frac{C(\alpha, \beta)}{a^{2}} F_{-}^{I}(x) F_{+}^{I}(x)(1+o(1)) .
$$

(Разделы (b), (c) в теореме 1.2 соответствуют классификации (а)(c), введенной ниже, перед теоремой 3.1.)

Естественно ожидать из соображений непрерывности, что утверждение п. (iii), (c) сохранится и при $\alpha=3$.

Отметим, что поправочный член в теореме 1.1 имеет относительно главной части порядок малости $1 / x$. В теореме 1.2 этот порядок составляет $\max \left(F_{-}^{I}(x), F_{+}^{I}(x)\right) \gg 1 / x, x \rightarrow \infty$.

При изучении асимптотики $\mathbf{P}(S \geqslant x)$ мы будем использовать факторизационное тождество

$$
\mathbf{E} e^{i \lambda S}=\frac{1-p}{1-\mathbf{E}\left(e^{i \lambda \chi_{+}} ; \eta_{+}<\infty\right)}=\frac{1-p}{1-p \mathbf{E} e^{i \lambda \chi}},
$$

где $\chi$ есть случайная величина с распределением

$$
\mathbf{P}(\chi \geqslant x)=\mathbf{P}\left(\chi_{+} \geqslant x \mid \eta_{+}<\infty\right),
$$

и

$$
p:=\mathbf{P}\left(\eta_{+}<\infty\right)=\mathbf{P}(S>0) .
$$

Из (1.8) следует, что

$$
\mathbf{P}(S \geqslant x)=(1-p) \sum_{n=0}^{\infty} p^{n} \mathbf{P}\left(X_{n} \geqslant x\right),
$$

где $X_{0}=0, X_{n}:=\sum_{j=1}^{n} \chi_{j}$ и $\chi_{j}, j=1,2, \ldots$, - независимые копии $\chi$. Из соотношения (1.9) видно, что для более полного знания асимптотики $\mathbf{P}(S \geqslant x)$ при $x \rightarrow \infty$ мы должны более полно изучить асимптотику $\mathbf{P}(\chi \geqslant x)$ и найти асимптотику второго порядка вероятности $\mathbf{P}\left(X_{n} \geqslant x\right)$ при $n \geqslant 2$. Этим двум задачам будут посвящены последующие два раздела: п. 2 и п. 3. Доказательство основной теоремы 1.2 приведено 
в п. 4. В п. 5 полученные результаты распространены на обобщенные процессы восстановления.

Результаты работы носят «теоретический» характер. Однако мотивированы они были главным образом приложениями. Автору не раз приходилось слышать, что, например, при не очень больших $x$ приближения первого порядка (1.1) не всегда оказываются удовлетворительными.

В случае конечной дисперсии аппроксимация второго порядка (1.3) использовалась в [7] в задаче страхования с кредитованием. Аналогичным образом результаты теоремы 1.2 могут быть использованы в той же задаче в случае бесконечной дисперсии.

2. Уточнения теоремы восстановления и асимптотик распределений первой положительной и первой отрицательной сумм. Пусть $\zeta, \zeta_{1}, \zeta_{2}, \ldots$ - неотрицательные одинаково распределенные независимые случайные величины $a_{\zeta}:=\mathbf{E} \zeta<\infty, F_{\zeta}(t):=\mathbf{P}(\zeta \geqslant t)$,

$$
Z_{0}=0, \quad Z_{n}:=\sum_{j=1}^{n} \zeta_{j}, \quad n \geqslant 1, \quad H(t):=\sum_{n=0}^{\infty} \mathbf{P}\left(Z_{n}<t\right) .
$$

В дальнейшем мы будем различать арифметические и неарифметические случайные величины; при этом неарифметичность мы будем понимать в широком смысле: не существует такого числа $d$, что все возможные значения случайной величины кратны $d$.

Теорема 2.1. Пусть $\zeta_{j}$ неарифметичны. Тогда справедливы следующие утверждения.

1. Если $b_{\zeta}:=\mathbf{E} \zeta^{2}<\infty$, mо при $t \rightarrow \infty$

$$
H(t)=\frac{t}{a_{\zeta}}+\frac{b_{\zeta}}{2 a_{\zeta}^{2}}+o(1)
$$

2. Ecлu $b_{\zeta}=\infty u F_{\zeta}^{I}(t):=\int_{t}^{\infty} F_{\zeta}(u) d u \in \mathscr{R}_{\gamma}, \gamma \in(0,1)$, mo npu $t \rightarrow \infty$

$$
H(t)=\frac{t}{a_{\zeta}}+\frac{t F_{\zeta}^{I}(t)}{(1-\gamma) a_{\zeta}^{2}}(1+o(1))
$$

Если $\zeta_{j}$ арифметичнь, то при иельх $t$ утверждения теоремьл сохранятся, но $b_{\zeta}$ в (2.1) следует заменить на $b_{\zeta}+a_{\zeta}$.

Д о к а з а т е л с с в о. Утверждение теоремы в случае $b_{\zeta}<\infty$ хорошо известно (см., например, $[4$, гл. XI], $[5$, гл. X]). Тем не менее ради цельности изложения мы приведем здесь полное доказательство теоремы, в котором существенное различие между случаями $b_{\zeta}<\infty$ и $b_{\zeta}=\infty$ отсутствует.

Обозначим

$$
\eta(t):=\min \left\{k: Z_{k} \geqslant t\right\}, \quad \chi(t):=Z_{\eta(t)}-t .
$$


Тогда для неотрицательных $\zeta$ в силу тождества Вальда (см., например, $[5$, гл. Х])

$$
H(t)=\mathbf{E} \eta(t)=\frac{t+\mathbf{E} \chi(t)}{a_{\zeta}},
$$

где

так что

$$
\mathbf{P}(\chi(t) \geqslant u)=\int_{0}^{t} d H(v) \mathbf{P}(\zeta \geqslant t-v+u),
$$

$$
\mathbf{E} \chi(t)=\int_{0}^{t} d H(v) F_{\zeta}^{I}(t-v) .
$$

В силу основной теоремы восстановления для неарифметических $\zeta$ имеем $H(v+\Delta)-H(v) \sim \Delta / a_{\zeta}$ при $v \rightarrow \infty$ и любом фиксированном $\Delta>0$. Отсюда и из (2.4) следует, что при $t \rightarrow \infty$

$$
\mathbf{E} \chi(t) \sim \frac{1}{a_{\zeta}} \int_{0}^{t} F_{\zeta}^{I}(u) d u \rightarrow \frac{b_{\zeta}}{2 a_{\zeta}} .
$$

Если $b_{\zeta}<\infty$, то отсюда следует (2.1). Если $b_{\zeta}=\infty$ и $F_{\zeta}^{I}(t) \in \mathscr{R}_{\gamma}, \gamma \in$ $(0,1)$, то из равенства $(2.3)$ и первого соотношения в $(2.5)$ получаем $(2.2)$ (о свойствах п.м.ф. и интегралов от них см., например, [3], [6]).

Доказательство в случае арифметических $\zeta_{j}$ вполне аналогично (см. $[4$, гл. Х]). Теорема доказана.

Рассмотрим теперь асимптотику распределения первой отрицательной суммы $\chi_{-}$.

О п р е д е л е н и е 2.1. Функция $F(t)$ называется асимптотически локально постоянной или просто локально постоянной (л.п.), если при $t \rightarrow \infty$ и любом фиксированном $c$

$$
\frac{F(t+c)}{F(t)} \rightarrow 1
$$

Нам понадобится следующее утверждение. Положим

$$
\zeta:=-\chi_{-} \text {. }
$$

Лемма 2.1. Пусть $a=-\mathbf{E} \xi>0$. Тогда:

(i) вьлолнено равенство

$$
a_{\zeta}:=\mathbf{E} \zeta=\frac{a}{1-p}
$$

(ii) $n p u$ всех $t>0$

$$
1 \leqslant \frac{\mathbf{P}(\zeta \geqslant t)}{F_{-}(t)} \leqslant \frac{1}{1-p} ;
$$

(iii) если $F_{-}$- л.n., mо при $t \rightarrow \infty$

$$
\mathbf{P}(\zeta \geqslant t) \sim \frac{F_{-}(t)}{1-p} .
$$


Следствие 2.1. 1) $b_{\zeta}=\mathbf{E} \zeta^{2}<\infty$ тогда и только тогда, когда $\mathbf{E} \xi_{-}^{2}<\infty$.

2) Если $F_{-}-$л.n. $u F_{-}^{I}(t) \in \mathscr{R}_{\gamma}$, mo $F_{\zeta}^{I}=F_{-}^{I}(t) /(1-p) \in \mathscr{R}_{\gamma}$.

Д о к а з а т ель с т в о ле м м ы 2.1. Утверждения леммы 2.1 известны (см., например, [5, гл. XII]). Однако их доказательство не занимает много места, и мы для полноты изложения его приведем. Обозначим $\mathfrak{f}(\lambda)=\mathbf{E} e^{i \lambda \xi}$ и воспользуемся следующим факторизационным тождеством, справедливым при $a>0$ :

$$
1-\mathfrak{f}(\lambda)=\left(1-\mathbf{E}\left(e^{i \lambda \chi_{+}} ; \eta_{+}<\infty\right)\right)\left(1-\mathbf{E} e^{i \lambda \chi_{-}}\right) .
$$

Это тождество можно переписать в виде

$$
1-\mathfrak{f}(\lambda)=\left(1-p \mathbf{E} e^{i \lambda \chi}\right)\left(1-\mathbf{E} e^{i \lambda \chi_{-}}\right) .
$$

Если поделить обе части тождества (2.10) на $\lambda$ и устремить $\lambda$ к 0, то мы получим (2.6). Из (2.10), (1.8) получаем

$$
\begin{gathered}
1-\mathbf{E} e^{i \lambda \chi_{-}}=\frac{\mathbf{E} e^{i \lambda S}}{1-p}(1-\mathfrak{f}(\lambda)) \\
\mathbf{P}(\zeta \geqslant t)=\mathbf{P}\left(\chi_{-} \leqslant-t\right)=\frac{1}{1-p} \int_{0}^{\infty} \mathbf{P}(S \in d u) \mathbf{P}(\xi \leqslant-t-u) \\
=\frac{1}{1-p} \int_{0}^{\infty} \mathbf{P}(S \in d u) F_{-}(t+u) .
\end{gathered}
$$

Отсюда следует, что при всех $t>0(\mathbf{P}(S=0)=1-p)$ справедливо (2.7). Кроме того, если $F_{-}$- л.п., то из (2.11) получаем (2.8). Лемма 2.1 доказана.

Следующим шагом при изучении асимптотики $\mathbf{P}(S \geqslant x)$ является описание асимптотики функции

$$
U(t):=\mathbf{P}(\chi \geqslant t) .
$$

Теорема 2.2. Пусть $a>0$ и распределение $\xi$ неарифметично. Тогда:

(i) если $\mathbf{E} \xi_{-}^{2}<\infty$, mo при $t \rightarrow \infty$

$$
U(t)=\frac{(1-p) F_{+}^{I}(t)}{p a}+\frac{b_{\zeta}(1-p)^{2} F_{+}(t)}{2 p a^{2}}+o\left(F_{+}(t)\right) ;
$$

(ii) если выполнено условие $\left[\mathbf{R}_{\beta}^{\alpha}\right]$, то при $t \rightarrow \infty$

$$
U(t)=\frac{1-p}{p a} F_{+}^{I}(t)+\frac{1-p}{p a^{2}} C(\alpha, \beta) F_{+}^{I}(t) F_{-}^{I}(t)(1+o(1)),
$$

где $C(\alpha, \beta)=(\alpha-1) \int_{1}^{\infty} s^{-\alpha}(s-1)^{1-\beta} d s$.

Если распределение $\xi$ арифметично, то при чельх $t$ утверждения теоремь сохранятся, но коэффициент $b_{\zeta}$ в (2.12) следует заменить на $b_{\zeta}+a_{\zeta}$. 
Д о к а з а т е л ь с т в о. В силу тождества (2.10) имеем

$$
p U(t)=p \mathbf{P}(\chi \geqslant t)=\int_{0}^{\infty} d H(u) F_{+}(t+u)=-\int_{t}^{\infty} H(v-t) d F_{+}(v) .
$$

Если $\mathbf{E} \xi_{-}^{2}<\infty$, то $b_{\zeta}<\infty$ в силу следствия 2.1 и по теореме 2.1 и лемме 2.1

$$
H(t)=\frac{t(1-p)}{a}+\frac{b_{\zeta}(1-p)^{2}}{2 a^{2}}+o(1) \quad \text { при } t \rightarrow \infty .
$$

Стало быть,

$$
p U(t)=-\frac{1-p}{a} \int_{t}^{\infty}(v-t) d F_{+}(v)+\frac{b_{\zeta}(1-p)^{2}}{2 a^{2}} F_{+}(t)+o\left(F_{+}(t)\right),
$$

где $-\int_{t}^{\infty}(v-t) d F_{+}(v)=F_{+}^{I}(t)$.

Это доказывает (2.12).

Если $\mathbf{E} \xi_{-}^{2}=\infty$, то по условиям теоремы $2.2 F_{-}-$л.п. и $F_{-}^{I} \in \mathscr{R}_{\gamma}$ при $\gamma=\beta-1$. В силу теоремы 2.1 и леммы $2.1 F_{\zeta}^{I}(t) \sim(1-p)^{-1} F_{-}^{I}(t)$ при $t \rightarrow \infty$ и

$$
H(t)=\frac{t(1-p)}{a}+\frac{t F_{-}^{I}(t)(1-p)}{a^{2}(1-\gamma)}(1+o(1))
$$

Полагая $Q(t):=t F_{-}^{I}(t)$, мы в силу $(2.13)$ получим

$$
p U(t)=\frac{1-p}{a} F_{+}^{I}(t)-\frac{1-p}{(1-\gamma) a^{2}} \int_{t}^{\infty} Q(v-t) d F_{+}(v) .
$$

Интегрируя по частям, находим

$$
\begin{aligned}
& -\int_{t}^{\infty} Q(v-t) d F_{+}(v)=\int_{t}^{\infty} F_{+}(v) d_{v} Q(v-t) \\
& \quad=\int_{t}^{\infty} F_{+}(v) F_{-}^{I}(v-t) d v-\int_{t}^{\infty} F_{+}(v)(v-t) F_{-}(v-t) d v
\end{aligned}
$$

Делая замену переменных $v=s t$, мы получим для первого интеграла в правой части (2.15) значение

$$
t F_{+}(t) F_{-}^{I}(t) \int_{1}^{\infty} \frac{F_{+}(s t)}{F_{+}(t)} \frac{F_{-}^{I}(t(s-1))}{F_{-}^{I}(t)} d s
$$

где подынтегральная функция для каждого $s$ сходится при $t \rightarrow \infty$ к $s^{-\alpha}(s-1)^{-\gamma}, \gamma=\beta-1$. Кроме того, в силу свойств п.м.ф. (см., например, [2], [6]) подынтегральная функция для заданного $\varepsilon>0$ при всех $s>1$ и всех достаточно больших $t$ не превосходит $c s^{-\alpha+\varepsilon}(s-1)^{-\gamma+\varepsilon}$, 
$c=$ const. Так как $\alpha>1$, то при $\varepsilon<\gamma / 2$ мы получаем, что подынтегральная функция в (2.16) допускает интегрируемую мажоранту и, стало быть,

$$
\int_{t}^{\infty} F_{+}(v) F_{-}^{I}(v-t) d v \sim C t F_{+}(t) F_{-}^{I}(t), \quad C=\int_{1}^{\infty} s^{-\alpha}(s-1)^{-\gamma} d s .
$$

Заметим теперь, что $t F_{-}(t) \sim(\beta-1) F_{-}^{I}(t)$ и, следовательно, второй интеграл в правой части $(2.15)$ имеет с точностью до множителя $(\beta-1)$ ту же асимптотику, что и первый. Поэтому при $t \rightarrow \infty$

$$
-\int_{t}^{\infty} Q(v-t) d F_{+}(v) \sim(2-\beta) C t F_{+}(t) F_{-}^{I}(t) .
$$

Так как $t F_{+}(t) \sim(\alpha-1) F_{+}^{I}(t)$, то вместе с (2.14) это доказывает второе утверждение теоремы.

В арифметическом случае изменения в доказательстве сводятся лишь к замене коэффициента $b_{\zeta}$ на $b_{\zeta}+a_{\zeta}$ в соответствии с теоремой 2.1 . Теорема 2.2 доказана.

3. Аппроксимация второго порядка вероятностей больших уклонений сумм неотрицательных случайных величин, не имеющих среднего. Обратимся теперь к представлению (1.9) и уточним асимптотику $\mathbf{P}\left(X_{n} \geqslant x\right), n \geqslant 2$, при $x \rightarrow \infty$, используя асимптотические разложения для $U(x)=\mathbf{P}(\chi \geqslant x)$, полученные в теореме 2.2 .

Отметим, что соотношение (1.9) можно записать также в виде

$$
\mathbf{P}(S \geqslant x)=\mathbf{P}\left(X_{\tau} \geqslant x\right),
$$

где $\tau$ не зависит от $\left\{X_{n}\right\}, \mathbf{P}(\tau=n)=(1-p) p^{n}, n=0,1, \ldots$.

Аппроксимация $\mathbf{P}\left(S_{n} \geqslant x\right)$ второго и более высоких порядков при $x \rightarrow \infty$, когда существует $\mathbf{E} \xi=0$, а $F_{+}(x)$ есть п.м.ф., изучена весьма полно в [3]. Если $\mathbf{E} \xi^{2}<\infty$, то при некоторых предположениях на гладкость $F_{+}(x)$ при $x \rightarrow \infty$ выполняется

$$
\mathbf{P}\left(S_{n} \geqslant x\right)=n F_{+}(x)\left[1+\frac{\alpha(\alpha+1)(n-1) \mathbf{E} \xi^{2}}{2 x^{2}}(1+o(1))\right]
$$

равномерно по $n \leqslant c x^{2} / \ln x$ при некотором $c>0$ и $x \rightarrow \infty$, где $-\alpha-$ степенной показатель п.м.ф. $F_{+}(x)$.

Если $\mathbf{E} \xi$ не существует, то природа второго члена асимптотики для $\mathbf{P}\left(S_{n} \geqslant x\right)$ существенно меняется, как это видно из теоремы 3.1 , приведенной ниже.

Как уже отмечалось, мы будем изучать в этом пункте асимптотику $\mathbf{P}\left(X_{n} \geqslant x\right), n \geqslant 2$, для сумм $X_{n}=\sum_{j=1}^{n} \chi_{j}$, где в соответствии с теоремой 2.2 хвост $U(x)=\mathbf{P}(\chi \geqslant x)$ распределения $\chi$ представим при $x \rightarrow \infty$ в виде

$$
U(x)=V(x)+w(x)(1+o(1)),
$$


где

1) $V(x)=\int_{x}^{\infty} v(t) d t$, функция $v \in \mathscr{R}_{\alpha}, \alpha>1$, монотонно убывает, так что

$$
V(x) \sim \frac{v(x) x}{\alpha-1} \quad \text { при } x \rightarrow \infty, \quad V \in \mathscr{R}_{\alpha-1} ;
$$

2) функция $w \in \mathscr{R}_{\gamma}$ монотонно убывает, $\gamma>\alpha-1$.

Согласно теореме 2.2 , при выполнении $\left[\mathbf{R}_{<\infty}^{\alpha}\right], \alpha \in(0,1)$, в (3.1) имеем

$$
V(x)=\frac{(1-p) F_{+}^{I}(x)}{p a}, \quad w(x)=\frac{b_{\zeta}(1-p)^{2} F_{+}(x)}{2 p a^{2}},
$$

так что $\gamma=\alpha$.

При выполнении $\left[\mathbf{R}_{\beta}^{\alpha}\right], \alpha>1, \beta \in(0,1)$, функция $V(x)$ сохраняет прежнее значение,

$$
w(x)=\frac{1-p}{p a^{2}} C(\alpha, \beta) F_{+}^{I}(x) F_{-}^{I}(x),
$$

так что $\gamma=\alpha+\beta-2>\alpha-1, \alpha>1$ - любое число.

Положим

$$
\Pi:=n U(x) \quad(\sim n V(x) \text { при } x \rightarrow \infty) .
$$

Из теоремы 2.6.1 в [3] вытекает следующее утверждение. Если $U(x) \in$ $\mathscr{R}_{\alpha-1}, \alpha \in(1,2)$, то существует функция $\theta(t) \downarrow 0, t \downarrow 0$, такая, что

$$
\left|\frac{\mathbf{P}\left(X_{n} \geqslant x\right)}{\Pi}-1\right|<\theta(\Pi) .
$$

Если существует $a_{\chi}:=\mathbf{E} \chi<\infty$, то обозначим

$$
\Pi_{1}:=n U\left(x-a_{\chi} n\right)
$$

Если, кроме того, $U \in \mathscr{R}_{\alpha-1}, \alpha \in(2,3)$ или $\alpha>3$, то из теорем 3.4.1, 4.4 .1 в [3] вытекает, что существует функция $\theta(t) \downarrow 0, t \downarrow 0$, такая, что

$$
\left|\frac{\mathbf{P}\left(X_{n} \geqslant x\right)}{\Pi_{1}}-1\right|<\theta\left(\Pi_{1}\right) .
$$

В дальнейшем нам понадобятся эти утверждения лишь для $n=o(x)$ при $x \rightarrow \infty$. Можно считать, например, что везде в дальнейшем $n<x^{\rho}$, где $\rho<\min (1 / 2,(\alpha-1) / 2)$ фиксировано. (На самом деле нам достаточно рассматривать $n<c \ln x, c=$ const; см. п. 4.) Для таких $n$ и $x \rightarrow \infty$ выполняется $\Pi_{1} \rightarrow 0$ (если $\left.a_{\chi}<\infty\right)$ и функции П и $\Pi_{1}$ асимптотически эквивалентны:

$$
\left.\Pi \sim \Pi_{1} \quad \text { при } \Pi_{1} \rightarrow 0 \quad \text { (или при } \Pi \rightarrow 0, n=o(x)\right) .
$$

Поэтому везде в дальнейшем (в тех случаях, когда $a_{\chi}<\infty$ ) мы для единообразия вместо $\Pi_{1}$ будем писать $\Pi$, имея в виду, что $n=o(x)$. 
Соотношения (3.3), (3.4) при $\Pi \rightarrow 0$ дают аппроксимаиию первого порядка для функции

$$
U_{n}(x)=\mathbf{P}\left(X_{n} \geqslant x\right) .
$$

Эта аппроксимация является равномерной по всем $n$ и $x$ таким, что $n U(x)=\Pi \rightarrow 0$; мы будем записывать ее в виде

$$
U_{n}(x)=\Pi(1+\bar{o}(1))
$$

Равномерность означает, что для заданного $\varepsilon>0$ найдется $\delta=\delta(\varepsilon)$ такое, что

$$
\left|\frac{U_{n}(x)}{\Pi}-1\right|<\varepsilon
$$

$n p u$ всех $n$ и $x$ таких, что $\Pi<\delta$. Равномерность такого вида будет иметь место во всех соотношениях этого пункта, например, в соотношениях $U(x)=V(x)(1+\bar{o}(1))($ так как $\Pi \rightarrow 0$ влечет за собой $x \rightarrow \infty)$,

$$
\left|\frac{U_{n}(t x)}{\Pi}-t^{1-\alpha}\right|=\bar{o}(1), \quad t>0,
$$

и др. При этом в силу свойств п.м.ф. (см., например, [3], [6]) сходимость в (3.6) будет также равномерной и по $t$ из любого отрезка $\left[t_{1}, t_{2}\right], t_{1}>0$.

Символ $\bar{O}(1)$ будет иметь аналогичный смысл равномерности: мы будем писать $\varphi(x, n)=\bar{O}(1)$, если найдется постоянная $c$ такая, что $\varphi(x, n) \leqslant c$ для всех $n$ и $x$, для которых П (или $\Pi_{1}$ ) достаточно мало. Символы $\bar{o}(\varphi(x, n))$ и $\bar{O}(\varphi(x, n))$ для $\varphi(x, n)>0$ означают соответственно $\varphi(x, n) \bar{o}(1)$ и $\varphi(x, n) \bar{O}(1)$.

Рассмотрим следующие три непересекающиеся возможности.

(a) $\alpha \in(1,2), w(x)=O\left(V^{2}(x)\right)$ при $x \rightarrow \infty$. Этот случай реализуется при условии $\left[\mathbf{R}_{<\infty}^{\alpha}\right], \alpha \in(1,2)$, а также при условиях $\left[\mathbf{R}_{\beta}^{\alpha}\right], \alpha \in(0,1)$, $F_{-}^{I}(x)=O\left(F_{+}^{I}(x)\right)$ при $x \rightarrow \infty$.

(b) $\alpha \in(1,2), w(x) \gg V^{2}(x)$ при $x \rightarrow \infty$. Этот случай имеет место при выполнении $\left[\mathbf{R}_{\beta}^{\alpha}\right], \alpha \in(1,2), F_{-}^{I}(x) \gg F_{+}^{I}(x)$ при $x \rightarrow \infty$.

(c) $\alpha>2$. Здесь всегда выполнено $w(x) \gg V^{2}(x)$. Этот случай возможен лишь при условии $\left[\mathbf{R}_{\beta}^{\alpha}\right]$.

Следующее утверждение посвящено аппроксимации второго порядка для $U_{n}(x)$ при выполнении (3.1).

Теорема 3.1.. Пусть вьлполнено (3.1) $u \Pi=n U(x) \rightarrow 0$. Тогда:

(a) если $\alpha \in(1,2), w(x)=O\left(V^{2}(x)\right)$ nри $x \rightarrow \infty$, mo

$$
U_{n}(x)=n U(x)+n(n-1) V^{2}(x) A(\alpha)(1+\bar{o}(1)),
$$

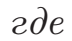

$$
A(\alpha)=2^{2(\alpha-1)}+(\alpha-1) \int_{0}^{1 / 2} s^{1-\alpha}(1-s)^{-\alpha} d s
$$


(b) если $\alpha \in(1,2), w(x) \gg V^{2}(x)$ при $x \rightarrow \infty$, mo

$$
U_{n}(x)=n U(x)+\bar{o}(n w(x))+\bar{O}\left(n(n-1) V^{2}(x)\right)
$$

(с) если $\alpha>2, \alpha \neq 3, n<x^{\rho}$, то при достаточно малом $\rho>0$

$$
U_{n}(x)=n U(x)+\bar{o}(n w(x)) .
$$

В [8] был получен следующий результат. Пусть $\mathscr{L}^{2}$ есть класс субэкспоненциальных распределений таких, что

$$
\lim _{x \rightarrow \infty} \frac{\mathbf{P}\left(S_{2} \geqslant x\right)-2 F_{+}(x)+F_{+}^{2}(x)}{F_{+}^{2}(x)}=0 .
$$

Тогда при любом фиксированном $n$

$$
\lim _{x \rightarrow \infty} \frac{\mathbf{P}\left(S_{n} \geqslant x\right)-n F_{+}(x)}{F_{+}^{2}(x)}=-\frac{n(n-1)}{2} .
$$

Сравнение с теоремой 3.1, (а) показывает, что $\mathscr{L}^{2} \cap \mathscr{R}_{\alpha-1}$ при $\alpha \in(1,2)$ есть класс распределений, для которых $A(\alpha)=-1 / 2$. Но $A(\alpha)>0$ при $\alpha \in(1,2)$, так что класс $\mathscr{L}^{2} \cap \mathscr{R}_{\alpha-1}$ при $\alpha \in(1,2)$ пуст.

Д ок аз а т ель с т в о т е о р м ы 3.1. (а) Положим

$$
\begin{gathered}
y=\frac{x}{r}, \quad r \in(2,3) ; \\
G_{n}=\left\{X_{n} \geqslant x\right\}, \quad B_{j}=\left\{\chi_{j}<y\right\}, \quad B=\bigcap_{j=1}^{n} B_{j} .
\end{gathered}
$$

Тогда, согласно теореме 2.2.1 в [3], для $\alpha \in(1,2)$

$$
\mathbf{P}\left(G_{n} B\right)=\bar{O}\left(\Pi^{r}\right)
$$

Поэтому

$$
\begin{aligned}
U_{n}(x) & =\mathbf{P}\left(G_{n}\right)=\mathbf{P}\left(G_{n} \bar{B}\right)+\mathbf{P}\left(G_{n} B\right)=\mathbf{P}\left(G_{n} \bar{B}\right)+\bar{O}\left(\Pi^{r}\right), \\
\mathbf{P}\left(G_{n} \bar{B}\right) & =\mathbf{P}\left(\bigcup_{j=1}^{n} G_{n} \bar{B}_{j}\right)=\Sigma_{1}-\Sigma_{2}+\bar{O}\left(\Pi^{3}\right),
\end{aligned}
$$

где

$$
\Sigma_{1}=\sum_{j=1}^{n} \mathbf{P}\left(G_{n} \bar{B}_{j}\right), \quad \Sigma_{2}=\sum_{i<j \leqslant n} \mathbf{P}\left(G_{n} \bar{B}_{i} \bar{B}_{j}\right) .
$$

Оценки слагаемых этих сумм мы выделим в отдельные утверждения. 
Лемма 3.1. При $\alpha \in(1,2), \Pi \rightarrow 0$ имеет место соотношение

$$
\begin{aligned}
\mathbf{P}\left(G_{n} \bar{B}_{j}\right)= & U(x)+(\alpha-1)(n-1) U^{2}(x) I(\alpha)\left(1+q_{2}(r)+\bar{o}(1)\right) \\
& +(n-1) V^{2}(x) \cdot 2^{2 \alpha-1}\left(1+q_{3}(r)+\bar{o}(1)\right)+\bar{o}(w(x)),
\end{aligned}
$$

где $q_{i}(r) \rightarrow 0$ при $r \downarrow 2, i=2,3$.

(Порядковая нумерация слагаемых $q_{i}(r), i=1, \ldots, 7$, устанавливается в доказательствах лемм 3.1, 3.2.)

Д ок а з а т е л ь с т о. Имеем

$$
P:=\mathbf{P}\left(G_{n} \bar{B}_{j}\right)=\mathbf{P}\left(G_{n} \bar{B}_{n}\right)=P_{1}+P_{2},
$$

где

$$
P_{1}=\mathbf{P}\left(X_{n-1} \geqslant x-y, \chi_{n} \geqslant y\right), \quad P_{2}=\mathbf{P}\left(X_{n-1}+\chi_{n} \geqslant x, X_{n-1}<x-y\right) .
$$

При $\Pi \rightarrow 0$ находим

$$
\begin{aligned}
P_{1} & =\mathbf{P}\left(X_{n-1} \geqslant x-y\right) \mathbf{P}\left(\chi_{n} \geqslant y\right)=(n-1) U(x-y) U(y)(1+\bar{o}(1)) \\
& =(n-1) r^{2(\alpha-1)}(r-1)^{1-\alpha} U^{2}(x)(1+\bar{o}(1)) \\
& =(n-1) \cdot 2^{2(\alpha-1)}\left(1+q_{1}(r)\right) U^{2}(x)(1+\bar{o}(1))
\end{aligned}
$$

где $q_{1}(r) \rightarrow 0$ при $r \downarrow 2$. Далее,

$$
P_{2} \equiv I_{0}^{x-y}:=-\int_{0}^{x-y} d U_{n-1}(t) U(x-t)=I_{0}^{\varepsilon x}+I_{\varepsilon x}^{x-y},
$$

где

$$
I_{0}^{\varepsilon x}=-\int_{0}^{\varepsilon x} d U_{n-1}(t) U(x-t)
$$

и $\varepsilon=\varepsilon(\Pi) \rightarrow 0$ при $\Pi \rightarrow 0$ достаточно медленно, так что $\varepsilon x \rightarrow \infty$. При $t<\varepsilon x$ имеем

$$
U(x-t)=V(x)+t v(x)(1+\bar{o}(1))+w(x)(1+\bar{o}(1)) .
$$

Поэтому

$$
\begin{aligned}
I_{0}^{\varepsilon x}= & V(x)\left[1-U_{n-1}(\varepsilon x)\right]+v(x)(1+\bar{o}(1)) \\
& \times \int_{0}^{\varepsilon x} t \mathbf{P}\left(\chi_{n-1} \in d t\right)+w(x)(1+\bar{o}(1)) .
\end{aligned}
$$

Здесь при $\varepsilon$, стремящемся к 0 настолько медленно, что $U(\varepsilon x)=$ $\varepsilon^{1-\alpha} U(x)(1+\bar{o}(1))$, выполняются соотношения

$$
\begin{aligned}
\int_{0}^{\varepsilon x} t \mathbf{P}\left(X_{n-1} \in d t\right) & =\frac{\alpha-1}{2-\alpha} \varepsilon x U_{n-1}(\varepsilon x)(1+\bar{o}(1)) \\
& =(n-1) \frac{\alpha-1}{2-\alpha} \varepsilon^{2-\alpha} x U(x)(1+\bar{o}(1)) .
\end{aligned}
$$


Стало быть,

$$
I_{0}^{\varepsilon x}=U(x)-V(x) U_{n-1}(\varepsilon x)+\bar{o}(w(x))+\varepsilon^{2-\alpha} \bar{O}\left(U^{2}(x)\right),
$$

где $\varepsilon^{2-\alpha} \bar{O}\left(U^{2}(x)\right)=\bar{o}\left(U^{2}(x)\right)$.

Рассмотрим теперь $I_{\varepsilon x}^{x-y}$, считая сначала, что $\varepsilon-$ малое фиксированное число. Так как $U_{n-1}(\varepsilon x)=\bar{o}(1)$ при $\Pi \rightarrow 0$, то

$$
I_{\varepsilon x}^{x-y}=-\int_{\varepsilon x}^{x-y} d U_{n-1}(t) V(x-t)+\bar{o}(w(x)) .
$$

Интегрируя по частям, находим

$$
\begin{aligned}
I_{\varepsilon x}^{x-y}= & -\left.U_{n-1}(t) V(x-t)\right|_{\varepsilon x} ^{x-y}+\int_{\varepsilon x}^{x-y} U_{n-1}(t) v(x-t) d t+\bar{o}(w(x)) \\
= & U_{n-1}(\varepsilon x) V(x)+U_{n-1}(\varepsilon x)[V(x(1-\varepsilon))-V(x)]-U_{n-1}(x-y) V(y) \\
& +x \int_{\varepsilon}^{1-1 / r} U_{n-1}(x s) v(x(1-s)) d s+\bar{o}(w(x)) .
\end{aligned}
$$

Так как $U_{n-1}(x s)=(n-1) s^{1-\alpha} U(x)(1+\bar{o}(1)), v(x(1-s))=(1-s)^{-\alpha} v(x) \times$ $(1+\bar{o}(1))$ равномерно по $s \in[\varepsilon, 1-1 / r]$, то здесь

$$
\begin{aligned}
x \int_{\varepsilon}^{1-1 / r} U_{n}(x s) v(x(1-s)) d s= & (n-1) x v(x) U(x) \\
& \times \int_{\varepsilon}^{1-1 / r} s^{1-\alpha}(1-s)^{-\alpha} d s(1+\bar{o}(1))
\end{aligned}
$$

при этом $x v(x)=(\alpha-1) V(x)(1+\bar{o}(1))=(\alpha-1) U(x)(1+\bar{o}(1))$,

$$
\int_{\varepsilon}^{1-1 / r} s^{1-\alpha}(1-s)^{-\alpha} d s=I(\alpha)-p_{1}(\varepsilon)+q_{2}(r),
$$

где $p_{1}(\varepsilon) \rightarrow 0$ при $\varepsilon \rightarrow 0$ и $q_{2}(r) \rightarrow 0$ при $r \downarrow 2$.

Кроме того,

$$
\begin{aligned}
U_{n-1}(x-y) V(y) & =(n-1) V^{2}(x) r^{2(\alpha-1)}(1-r)^{1-\alpha}(1+\bar{o}(1)) \\
& =(n-1) V^{2}(x) \cdot 2^{2(\alpha-1)}\left(1+q_{3}(r)\right)(1+\bar{o}(1)),
\end{aligned}
$$

где $q_{3}(r) \rightarrow 0$ при $r \downarrow 2$ и

$$
U_{n-1}(\varepsilon x)[V(x(1-\varepsilon))-V(x)]=(n-1) \varepsilon^{2-\alpha} U(x) x v(x)(1+\bar{o}(1))
$$

при $\varepsilon$, стремящемся к 0 достаточно медленно. Ясно, что другие соотношения, описывающие поведение $I_{\varepsilon x}^{x-y}$, также останутся справедливыми, 
если $\varepsilon=\varepsilon(\Pi)$ стремится к 0 при $\Pi \rightarrow 0$ достаточно медленно. Поэтому в результате мы получаем

$$
\begin{aligned}
I_{\varepsilon x}^{x-y}= & U_{n-1}(\varepsilon x) V(x)+(n-1)(\alpha-1) U^{2}(x)\left[I(\alpha)-p_{1}(\varepsilon)+q_{2}(r)\right](1+\bar{o}(1)) \\
& +(n-1) V^{2}(x) \cdot 2^{2(\alpha-1)}\left(1+q_{3}(r)\right)(1+\bar{o}(1)) \\
& +(n-1) V^{2}(x) \bar{o}(1)+\bar{o}(w(x)) .
\end{aligned}
$$

Складывая это с (3.17), получим

$$
\begin{aligned}
P_{2} \equiv I_{0}^{x-y}= & U(x)+(n-1)(\alpha-1) U^{2}(x)\left[I(\alpha)-p_{1}(\varepsilon)+q_{2}(r)\right](1+\bar{o}(1)) \\
& +(n-1) V^{2}(x) \cdot 2^{2(\alpha-1)}\left(1+q_{3}(r)\right)(1+\bar{o}(1))+\bar{o}(w(x)) .
\end{aligned}
$$

Учитывая (3.15), находим, что $P=P_{1}+P_{2}$ допускает такую же запись, но с заменой в правой части множителя $2^{2(\alpha-1)}$ на $2^{2 \alpha-1}$. Кроме того, слагаемое $q_{3}(r)$ будет иметь несколько иной смысл, но свойство $q_{3}(r) \rightarrow 0$ при $r \downarrow 2$ сохранится. Лемма 3.1 доказана.

Лемма 3.2. При $\Pi \rightarrow 0, i \neq j$ выполняются оченки

$$
\begin{aligned}
2^{2(\alpha-1)} U^{2}(x) & \left(1+q_{4}(r)+\bar{o}(1)\right) \\
& \left.\geqslant 2^{2(\alpha-1)} U^{2}(x)\left(1-q_{5}(r)+q_{n} \bar{B}_{i} \bar{B}_{j}\right)+\bar{o}(1)\right),
\end{aligned}
$$

где $q_{l}(r) \rightarrow 0$ при $r \downarrow 2, l=4,5$; оченки в (3.18) от $i$ и $j$ не зависят.

Д о к а з а т е л в с т в о. Имеем

$$
\begin{aligned}
\mathbf{P}\left(G_{n} \bar{B}_{i} \bar{B}_{j}\right) & =\mathbf{P}\left(G_{n} \bar{B}_{n-1} \bar{B}_{n}\right)=\int_{y}^{\infty} \int_{y}^{\infty} d U(t) d U(s) U_{n-2}(x-t-s) \\
& =\int_{t \geqslant y, s \geqslant y, t+s \geqslant x} d U(t) d U(s)+\int_{T_{y}} d U(t) d U(s) U_{n-2}(x-t-s),
\end{aligned}
$$

где $T_{y}$ - треугольная область $\{(t, s): t \geqslant y, s \geqslant y, s+t<x\}$. Поэтому

$$
\mathbf{P}\left(G_{n} \bar{B}_{n-1} \bar{B}_{n}\right)=U^{2}(y)-\int_{T_{y}} d U(t) d U(s)\left[1-U_{n-2}(x-t-s)\right] \leqslant U^{2}(y) .
$$

Кроме того,

$$
U^{2}(y)=U^{2}(x) \cdot 2^{2(\alpha-1)}\left(1+q_{4}(r)+\bar{o}(1)\right) \quad \text { при } \Pi \rightarrow 0,
$$

где $q_{4}(r) \rightarrow 0$ при $r \downarrow 2$. Это доказывает первое неравенство в (3.18).

Далее, интеграл в (3.19) не превосходит

$$
\begin{aligned}
\int_{T_{y}} d U(t) d U(s) & \leqslant\left(\int_{y}^{x-y} d U(t)\right)^{2} \\
& =(U(y)-U(x-y))^{2}=U^{2}(x) q_{5}(r)(1+\bar{o}(1)),
\end{aligned}
$$


где

$$
q_{5}(r)=r^{\alpha-1}-\left(\frac{r-1}{r}\right)^{1-\alpha} \rightarrow 0 \quad \text { при } r \downarrow 2 .
$$

Сказанное доказывает второе неравенство в (3.18). Лемма 3.2 доказана.

Вернемся к доказательству п. (а) теоремы 3.1. В силу (3.12) и лемм $3.1,3.2$

$$
\begin{aligned}
U_{n}(x) & \leqslant n \mathbf{P}\left(G_{n} \bar{B}_{n}\right)-\frac{n(n-1)}{2} \mathbf{P}\left(G_{n} \bar{B}_{n-1} \bar{B}_{n}\right)+\bar{O}\left(\Pi^{r}\right) \\
& =n U(x)+n(n-1) U^{2}(x) A(\alpha)\left(1+q_{6}(r)+\bar{o}(1)\right)+\bar{o}(n w(x))+\bar{O}\left(\Pi^{r}\right),
\end{aligned}
$$

где $q_{6}(r) \rightarrow 0$ при $r \downarrow 2$,

$$
A(\alpha)=(\alpha-1) I(\alpha)+2^{2(\alpha-1)} .
$$

Так как

$$
w(x)=O\left(V^{2}(x)\right) \quad \text { при } x \rightarrow \infty,
$$

TO

$$
\varlimsup_{\Pi \rightarrow 0} \frac{U_{n}(x)-n U(x)}{n(n-1) V^{2}(x)} \leqslant A(\alpha)\left(1+q_{6}(r)\right) .
$$

Аналогично,

$$
\varliminf_{\Pi \rightarrow 0} \frac{U_{n}(x)-n U(x)}{n(n-1) V^{2}(x)} \geqslant A(\alpha)\left(1+q_{7}(r)\right), \quad q_{7}(r) \rightarrow 0 \quad \text { при } r \downarrow 2 .
$$

Левые части этих неравенств от $r$ не зависят. Правые части по форме зависят от $r$, но выбором $r>2$, достаточно близкого к 2 , могут быть сделаны сколь угодно близкими к $A(\alpha)$. Это возможно лишь в случае, когда существует

$$
\lim _{\Pi \rightarrow 0} \frac{U_{n}(x)-n U(x)}{n(n-1) V^{2}(x)}=A(\alpha) .
$$

Это доказывает первое утверждение теоремы.

(b) Рассмотрим теперь альтернативный к (3.20) случай $w(x) \gg$ $V^{2}(x)$ при $x \rightarrow \infty$. Тогда из лемм $3.1,3.2$ вытекает, что

$$
U_{n}(x)=n U(x)+\bar{o}(n w(x))+\bar{O}\left(n(n-1) V^{2}(x)\right)+\bar{O}\left(\Pi^{r}\right) .
$$

Соотношение (3.9) доказано.

(с) Пусть теперь $\alpha \in(2,3)$. В этом случае существует $a_{\chi}=\mathbf{E} \chi$, соотношение (3.3) заменяется на эквивалентное (при рассматриваемых значениях $n=o(x))$ соотношение (3.4). Воспользуемся опять соотношением (3.12), но теперь при $r=2$, в котором в обозначениях (3.13)

$$
\mathbf{P}\left(G_{n} \bar{B}\right)=\Sigma_{1}+\bar{O}\left(\Pi^{2}\right) .
$$

Для новой оценки $\Sigma_{1}$ нам понадобится следующая лемма. 
Лемма 3.3. При $\alpha \in(2,3), \Pi \rightarrow 0$ выполняется

$$
\mathbf{P}\left(G_{n} \bar{B}_{j}\right)=U(x)+\bar{O}(n v(x))+\bar{o}(w(x)) .
$$

Д о к а з а т е ль с т в о. Воспользуемся опять соотношениями (3.14), (3.15) при $r=2$. Из (3.15) вытекает, что

$$
P_{1}<c(n-1) U^{2}(x) .
$$

Остается оценить

$$
\begin{aligned}
P_{2} & =-\int_{0}^{x / 2} d U_{n-1}(t) U(x-t) \\
& =U(x)\left(1-U_{n-1}\left(\frac{x}{2}\right)\right)-\int_{0}^{x / 2} d U_{n-1}(t)[U(x-t)-U(x)],
\end{aligned}
$$

где

$$
U(x-t)-U(x) \leqslant c t v(x)+o(w(x)) \quad \text { при } t \in\left[0, \frac{x}{2}\right], x \rightarrow \infty .
$$

Поэтому интеграл в правой части (3.24) не превосходит $c v(x)(n-1) a_{\chi}+$ $o(w(x))=\bar{O}(n v(x))+\bar{o}(w(x))$. Так как $U_{n-1}(x / 2)=\bar{O}(\Pi)$ для $\alpha \in(2,3)$ (см. (3.4)), то в результате получаем

$$
P_{2}=U(x)+\bar{O}\left(n V^{2}(x)\right)+\bar{O}(n v(x))+\bar{o}(w(x)),
$$

что вместе с (3.23) и соотношением $V^{2}(x)=o(v(x))$ доказывает (3.22). Лемма 3.3 доказана.

Вернемся к доказательству п. (с) теоремы 3.1. Имеем в силу (3.21) и леммы 3.3

$$
U_{n}(x)=n U(x)+\bar{O}\left(n^{2} v(x)\right)+\bar{o}(n w(x))+\bar{O}\left(\Pi^{2}\right) .
$$

Здесь, очевидно,

$$
\Pi^{2} \ll n^{2} v(x) \quad \text { при } \alpha>2, \Pi \rightarrow 0 .
$$

Сравним теперь $n w(x)$ и $n^{2} v(x)$ или, что то же, $w(x)$ и $n v(x)$. При $n<x^{\rho}$ и достаточно малом $\rho$ имеем

$$
-\gamma=2-\alpha-\beta>\rho-\alpha \quad \text { при } \beta \in(1,2)
$$

и, стало быть, $n^{2} v(x) \ll n w(x)$ при $\Pi \rightarrow 0$. Таким образом, из (3.25) мы получаем (3.10).

Нам осталось рассмотреть случай $\alpha>3$. Если $\alpha>3$, то при $r=2$, $n<x^{\rho}$ по теореме 4.1.1 в [3] вместо (3.11) выполняется соотношение

$$
\mathbf{P}\left(G_{n} B\right)=\bar{O}\left(\Pi^{r_{1}}\right)
$$


при любом фиксированном $r_{1}<2$. Кроме того, сохраняются соотношение (3.4) и утверждение леммы 3.3. Поэтому справедливо соотношение $(3.25)$, в котором $\Pi^{2}$ надо заменить на $\Pi^{r_{1}}$. Ясно, что при $r_{1}$, достаточно близком к 2 , и достаточно малом $\rho$ все рассуждения, доказывающие (3.10) с помощью (3.25), при $\alpha>3$ сохранятся. Теорема 3.1 доказана.

4. Доказательство теоремы 1.2. Докажем сначала утверждение теоремы в терминах представления (3.1).

Теорема 4.1. Пусть выполнено (3.1). Тогда справедливь следующие утверждения.

(a) Eсли $\alpha \in(1,2), w(x)=O\left(V^{2}(x)\right)$ при $x \rightarrow \infty$, mо

$$
\mathbf{P}(S \geqslant x)=\frac{p V(x)}{1-p}+\frac{p w(x)}{1-p}(1+o(1))+\frac{2 p^{2} A(\alpha) V^{2}(x)}{(1-p)^{2}}(1+o(1))
$$

где $A(\alpha)$ определено в (3.8).

$(\mathrm{b}, \mathrm{c})$ Если вьполнено одно из следующих двух условий:

(b) $\alpha \in(1,2), w(x) \gg V^{2}(x)$ nри $x \rightarrow \infty$ или

(c) $\alpha>2, \alpha \neq 3$,

mo при $x \rightarrow \infty$

$$
\mathbf{P}(S \geqslant x)=\frac{p V(x)}{1-p}+\frac{p w(x)}{1-p}(1+o(1))
$$

Д о к а з а т е л ь с т в о. Воспользуемся равенством (1.9) и разделим сумму в (1.9) на две подсуммы: $\Sigma_{\mathrm{I}}:=\sum_{n \leqslant n_{x}}$ (по значениям $n \leqslant n_{x}$ ) и $\Sigma_{\mathrm{II}}:=\sum_{n>n_{x}}\left(\right.$ по $\left.n>n_{x}\right)$, где $n_{x}=[c \ln x], c=-2 \alpha / \ln p$. Тогда вторая подсумма не превосходит

$$
\frac{p^{-c \ln x}}{(1-p) p}=\frac{x^{-2 \alpha}}{(1-p) p}
$$

Оценим теперь $\Sigma_{\mathrm{I}}$.

Рассмотрим сначала случай (а): если $\alpha \in(1,2), w(x)=O\left(V^{2}(x)\right)$ при $x \rightarrow \infty$, то по теореме 3.1

$$
\begin{aligned}
\Sigma_{\mathrm{I}} & =(1-p) \sum_{n \leqslant n_{x}} p^{n} \mathbf{P}\left(X_{n} \geqslant x\right) \\
& =(1-p) U(x) \sum_{n \leqslant n_{x}} n p^{n}+(1-p) A(\alpha) V^{2}(x) \sum_{n \leqslant n_{x}} n(n-1) p^{n}(1+\bar{o}(1)),
\end{aligned}
$$

где $\bar{o}(1)$ есть функция двух переменных $n$ и $x$, которая равномерно по $n$ и $x$ сходится к 0 , если $\Pi=n V(x)$ сходится к нулю. Но П сходится к 
нулю, если $n \leqslant n_{x}$ и $x \rightarrow \infty$, так как $V(x) \ln x \rightarrow 0$ при $x \rightarrow \infty$. Поэтому, учитывая равенства

$$
\sum_{n=0}^{\infty} n p^{n}=\frac{p}{(1-p)^{2}}, \quad \sum_{n=0}^{\infty} n(n-1) p^{n}=\frac{2 p^{2}}{(1-p)^{3}}
$$

получим при $x \rightarrow \infty$

$$
\Sigma_{\mathrm{I}}=\frac{p U(x)}{1-p}+\frac{2 p^{2} A(\alpha) V^{2}(x)}{(1-p)^{2}}(1+\bar{o}(1))+\bar{O}\left(x^{-2 \alpha} \ln ^{2} x\right)
$$

Это доказывает (4.1).

Рассмотрим случай (b): $\alpha \in(1,2), w(x) \gg V^{2}(x)$ при $x \rightarrow \infty$. В этом случае по теореме 3.1 совершенно аналогично находим при $x \rightarrow \infty$

$$
\begin{aligned}
\Sigma_{\mathrm{I}} & =\frac{p U(x)}{1-p}+\bar{o}(w(x))+\bar{O}\left(V^{2}(x)\right)+\bar{O}\left(x^{-2 \alpha} \ln ^{2} x\right) \\
& =\frac{p V(x)}{1-p}+\frac{p w(x)}{1-p}(1+\bar{o}(1)) .
\end{aligned}
$$

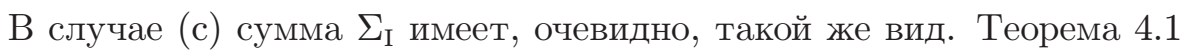
доказана.

Вернемся к теореме 1.2. Она вытекает из теорем 4.1, 2.2. Напомним, что при выполнении условия $\left[\mathbf{R}_{<\infty}^{\alpha}\right]$

$$
V(x)=\frac{(1-p) F_{+}^{I}(x)}{p a}, \quad w(x)=\frac{(1-p) b_{\zeta} F_{+}(x)}{2 p a^{2}} .
$$

При выполнении $\left[\mathbf{R}_{\beta}^{\alpha}\right]$ обозначение $V(x)$ сохраняет свой смысл,

$$
w(x)=\frac{1-p}{p a^{2}} C(\alpha, \beta) F_{-}(x) F_{+}(x)
$$

Если выполнено условие $\left[\mathbf{R}_{<\infty}^{\alpha}\right], \alpha \in(1,2)$, то $\alpha>2(\alpha-1)$ и, стало быть, $w(x) \ll V^{2}(x)$ при $x \rightarrow \infty$, так что следует воспользоваться равенством (4.1), в котором среднее слагаемое исчезает.

Если выполнено $\left[\mathbf{R}_{\beta}^{\alpha}\right], \alpha \in(1,2), F_{-}(x)=O\left(F_{+}(x)\right)$ при $x \rightarrow \infty$, то следует вновь воспользоваться равенством (4.1), но при новом значении $w$ (см. (4.5)), так что среднее слагаемое в (4.1) может быть сравнимым с последним.

Если выполнено $\left[\mathbf{R}_{\beta}^{\alpha}\right]$ и одно из условий (b) или (c), то следует воспользоваться равенством (4.2). Теорема 1.2 доказана. 
5. Аппроксимация второго порядка для максимума обобщенных процессов восстановления. В этом пункте мы получим аналог теоремы 1.2 для обобщенных процессов восстановления.

Пусть $\tau, \tau_{1}, \tau_{2}, \ldots$ - последовательность положительных независимых одинаково распределенных случайных величин с конечным средним $a_{\tau}:=\mathbf{E} \tau$. Положим $T_{0}:=0$,

$$
T_{k}:=\tau_{1}+\cdots+\tau_{k}, \quad k=1,2, \ldots,
$$

и пусть

$$
\nu(t):=\sum_{k=1}^{\infty} \mathbf{1}\left(T_{k} \leqslant t\right), \quad t \geqslant 0,
$$

есть порожденный этой последовательностью (непрерывный справа) процесс восстановления. Заметим, что $\{\nu(t)<k\}=\left\{T_{k}>t\right\}$ и что $\nu(t)+1$ есть момент первого прохождения уровня $t$ случайным блужданием $\left\{T_{k} ; k \geqslant 0\right\}$ :

$$
\nu(t)+1=\min \left\{k \geqslant 0: T_{k}>t\right\} .
$$

Предположим далее, что

$$
S_{0}=0, \quad S_{k}=\xi_{1}+\cdots+\xi_{k}, \quad k \geqslant 1,
$$

есть независимое от $\left\{\tau_{i} ; i \geqslant 1\right\}$ случайное блуждание, порожденное последовательностью независимых одинаково распределенных случайных величин $\xi, \xi_{1}, \xi_{2}, \ldots$.

Процесс с непрерывным временем

$$
Z(t):=S_{\nu(t)}+q t, \quad t \geqslant 0,
$$

где $q$ - вещественное число, называется обобщенным проиессом восстановления с линейныл сносом $q$.

Обозначим

$$
\bar{Z}:=\sup _{t \geqslant 0} Z(t) .
$$

Задача об асимптотике $\mathbf{P}(\bar{Z} \geqslant x)$ при $x \rightarrow \infty$ во многом может быть сведена к задаче об асимптотике $\mathbf{P}(S \geqslant x)$ для максимумов $S=\sup _{k} S_{k}$ обычных случайных блужданий, рассмотренных в пп. 1-3. Это следует из того, что при $q \leqslant 0$

$$
\bar{Z}=\sup _{k \geqslant 0}\left(S_{k}+q T_{k}\right)=: Y
$$

а при $q>0$

$$
\bar{Z}=\sup _{k \geqslant 1}\left(S_{k-1}+q T_{k}\right)=q \tau_{1}+\sup _{k \geqslant 1}\left[S_{k-1}+q\left(T_{k}-\tau_{1}\right)\right] \stackrel{d}{=} q \tau+Y,
$$


где случайные величины $\tau$ и $Y$ независимы, $Y=\sup _{k \geqslant 0} Y_{k}$ для случайного блуждания $Y_{k}=\sum_{j=1}^{k} \eta_{j}$, порожденного независимыми одинаково распределенными случайными величинами $\eta_{j}=\xi_{j}+q \tau_{j}$. Из этих представлений в [3] (см. теорему 16.1.1) получены следующие асимптотические соотношения первого порядка.

Теорема 5.1. Пусть $a_{\eta}:=\mathbf{E} \eta=-a+q a_{\tau}<0$. Тогда справедливь следующие утверждения.

I. Eсли $q \leqslant 0$ u $F_{+} \in \mathscr{R}_{\alpha}$, mo

$$
\mathbf{P}(\bar{Z} \geqslant x) \sim \frac{1}{\left|a_{\eta}\right|} \int_{x}^{\infty} F_{+}(t) d t \sim \frac{x F_{+}(x)}{(\alpha-1)\left|a_{\eta}\right|} .
$$

II. Если $q>0$ и выполнено одно из следующих трех условий:

(i) $F_{+} \in \mathscr{R} u F_{\tau}(t)=o\left(F_{+}(t)\right) n p u t \rightarrow \infty$;

(ii) $F_{+} \in \mathscr{R}, F_{\tau} \in \mathscr{R}$;

(iii) $F_{\tau} \in \mathscr{R} u F_{+}(t)=o\left(F_{\tau}(t)\right)$ npu $t \rightarrow \infty$, mo

$$
\mathbf{P}(\bar{Z} \geqslant x) \sim \frac{1}{\left|a_{\eta}\right|} \int_{x}^{\infty}\left(F_{+}(t)+F_{\tau}\left(\frac{t}{q}\right)\right) d t .
$$

Заметим, что в случаях II(i) и II(iii) второе и первое слагаемое соответственно под интегралом в (5.4) становятся пренебрежимо малыми и их можно опустить.

Наибольший интерес в приложениях представляет случай $q \leqslant 0$. С другой стороны, формулировка результатов в случае $q>0$ наиболее громоздка. Поэтому для краткости мы ограничимся рассмотрением асимптотики $\mathbf{P}(\bar{Z} \geqslant x)$ при $q \leqslant 0$. Случай $q>0$ может быть рассмотрен совершенно аналогично.

Справедливо следующее утверждение, описывающее асимптотику второго порядка $\mathbf{P}(\bar{Z} \geqslant x)$ при $q \leqslant 0$. Обозначения $F_{ \pm}(x), \alpha, \beta$ попрежнему относятся к случайным величинам $\xi$.

Кроме того, мы будем использовать обозначения

$$
\begin{gathered}
\eta=\xi+q \tau, \quad \xi_{( \pm)}= \pm \max (0, \pm \xi), \quad \eta_{( \pm)}= \pm \max (0, \pm \eta) \\
\left(\text { так что } \xi=\xi_{(+)}+\xi_{(-)}, \eta=\eta_{(+)}+\xi_{(-)}\right) \\
F_{\eta+}(t)=\mathbf{P}(\eta \geqslant 1), \quad F_{\eta-}(t)=\mathbf{P}(\eta \leqslant-t) \quad \text { при } t>0 .
\end{gathered}
$$

Теорема 5.2. I. Если $q=0$, mо $\bar{Z}=Y=S$ и асимптотика $\mathbf{P}(\bar{Z} \geqslant x)$ описььвается теоремой 1.2 без каких-либо изменений.

II. Пусть $q<0$.

(i) Если вылолненьл условия $\left[\mathbf{R}_{<\infty}^{\alpha}\right], \alpha \in(1,2), \mathbf{E} \tau^{2}<\infty$, то $\mathbf{E} \eta_{(-)}^{2}<$ $\infty, F_{\eta+} \in \mathscr{R}_{\alpha}$ и вероятность $\mathbf{P}(\bar{Z} \geqslant x)=\mathbf{P}(Y \geqslant x)$ равна правой части соотношения (1.5), в которой а надо заменить на $\left|a_{\eta}\right|$. 
(ii) $\Pi$ псть $\max \left(\mathbf{E} \tau^{2}, \mathbf{E} \xi_{(-)}^{2}\right)=\infty, F_{+}(t) \in \mathscr{R}_{\alpha}, \alpha \in(1,2), F_{\eta_{-}}(t) \in$ $\mathscr{R}, F_{\eta-}(t)=O\left(F_{+}(t)\right)$ при $t \rightarrow \infty u-\beta_{\eta}$ есть степенной показатель n.м.ф. $F_{\eta-}(t), t \rightarrow \infty$. Тогда вероятность $\mathbf{P}(\bar{Z} \geqslant x)$ равна правой части в (1.6), в которой надо заменить а на $\left|a_{\eta}\right|, \beta$ на $\beta_{\eta}, F_{-}^{I}(x)$ на $F_{\eta-}^{I}(x)=$ $\int_{x}^{\infty} F_{\eta-}(t) d t$.

(iii) Пусть $\max \left(\mathbf{E} \tau^{2}, \mathbf{E} \xi_{(-)}^{2}\right)=\infty, F_{\eta-} \in \mathscr{R}_{\beta_{\eta}}, \beta_{\eta} \in(1,2)$ и вьлолнено одно из двух условий:

либо $F_{+} \in \mathscr{R}_{\alpha}, \alpha \in(1,2), F_{\eta-}(x) \gg F_{+}(x)$ при $x \rightarrow \infty$,

либо $F_{+} \in \mathscr{R}_{\alpha}, \alpha>2, \alpha \neq 3$.

Тогда вероятность $\mathbf{P}(\bar{Z} \geqslant x)$ равна правой части в (1.7), в которой надо заменить а на $\left|a_{\eta}\right|, \beta$ на $\beta_{\eta}, F_{-}^{I}(x)$ на $F_{\eta-}^{I}(x)$.

3 а м е ч а н и е. 5.1 По поводу условия $F_{\eta-} \in \mathscr{R}$ можно заметить, что оно выполнено очевидным образом в следующих случаях.

(a) $F_{-} \in \mathscr{R}_{\beta}, F_{\tau}(t):=\mathbf{P}(\tau \geqslant t)=o\left(F_{-}(t)\right)$ при $t \rightarrow \infty$.

Тогда $F_{\eta-}(t) \sim F_{-}(t)$ при $t \rightarrow \infty, \beta_{\eta}=\beta$.

(b) $F_{-} \in \mathscr{R}_{\beta}, F_{\tau} \in \mathscr{R}_{\beta}$.

Тогда $F_{\eta-}(t) \sim F_{-}(t)+F_{\tau}(t), \beta_{\eta}=\beta$.

(c) $F_{\tau} \in \mathscr{R}_{\beta_{\tau}}, F_{-}(t)=o\left(F_{\tau}(t)\right)$ при $t \rightarrow \infty$.

Тогда $F_{\eta-}(t) \sim F_{\tau}(t), \beta_{\eta}=\beta_{\tau}$.

Д о к а з а т е л ь с т в о т е о р е м ы 5.2. I. Первое утверждение теоремы очевидно.

II. Пусть $q<0$. Тогда $\bar{Z}=Y$ и чтобы воспользоваться теоремой 1.2 , надо найти асимптотику $F_{\eta+}(x)=\mathbf{P}(\eta \geqslant x), F_{\eta-}(x)=\mathbf{P}(\eta \leqslant-x)$ и $F_{\eta+}^{I}(x):=\int_{x}^{\infty} F_{\eta+}(t) d t$. Нетрудно видеть, что при $q<0, x \rightarrow \infty$

$$
F_{\eta+}(x)=\mathbf{P}(\xi+q \tau \geqslant x) \sim \mathbf{P}(\xi \geqslant x)=F_{+}(x) .
$$

Далее, для последовательности $\varepsilon=\varepsilon(x)$, при $x \rightarrow \infty$ сходящейся к 0 настолько медленно, что $\varepsilon x \rightarrow \infty$, имеем

$$
\begin{aligned}
F_{\eta+}^{I}(x) & =\int_{x}^{\infty} \mathbf{P}(\xi \geqslant v-q \tau) d v=\mathbf{E} F_{+}^{I}(x-q \tau) \\
& =F_{+}^{I}(x)+\mathbf{E}\left[\left(F_{+}^{I}(x-q \tau)-F_{+}^{I}(x)\right) ; \tau<\varepsilon x\right]+\theta F_{+}^{I}(x) \mathbf{P}(\tau \geqslant \varepsilon x),
\end{aligned}
$$

где $|\theta| \leqslant 1$ и по теореме о среднем

$$
\mathbf{E}\left[\left(F_{+}^{I}(x-q \tau)-F_{+}^{I}(x)\right) ; \tau<\varepsilon x\right]=-q \mathbf{E}\left[\tau F_{+}\left(x-\theta_{\tau} \varepsilon x\right) ; \tau<\varepsilon x\right],
$$

$\left|\theta_{\tau}\right| \leqslant 1, F_{+}(x+t) \sim F_{+}(x)$ равномерно по $t \in[0, \varepsilon x]$. Поэтому значение (5.6) асимптотически эквивалентно $-q F_{+}(x) \mathbf{E} \tau$. Кроме того, так как $\mathbf{E} \tau<\infty$, то последовательность $\varepsilon(x)$ можно выбрать настолько медленно сходящейся к нулю, что $\mathbf{P}(\tau \geqslant \varepsilon x)=o(1 / x)$. В результате мы получаем

$$
F_{\eta+}^{I}(x)=F_{+}^{I}(x)-q F_{+}(x) \mathbf{E} \tau+F_{+}^{I}(x) o\left(\frac{1}{x}\right) .
$$


Отметим далее, что из неравенств

$$
\xi_{(-)}+q \tau \leqslant \eta_{(-)}=\min (0, \xi+q \tau) \leqslant \xi_{(-)}
$$

и

$$
\mathbf{P}\left(\eta_{(-)}<-v\right) \geqslant \mathbf{P}(\xi<0) \mathbf{P}\left(\tau>\frac{v}{q}\right)
$$

вытекает, что $\mathbf{E} \eta_{(-)}^{2}<\infty$ тогда и только тогда, когда $\mathbf{E} \xi_{(-)}^{2}<\infty, \mathbf{E} \tau^{2}<$ $\infty$.

Мы можем теперь перейти к доказательству подраздела (i) второго утверждения теоремы 5.2 .

(i) Пусть $\mathbf{E} \tau^{2}<\infty$ и $\xi$ удовлетворяет условию $\left[\mathbf{R}_{<\infty}^{\alpha}\right], \alpha \in(0,1)$. Тогда $\mathbf{E} \eta_{(-)}^{2}<\infty$ и нужное нам утверждение II(i) вытекает из (5.5), (5.7) и утверждения (i) теоремы 1.2. При этом надо учесть, что в (5.7)

$$
F_{+}(t)=o\left(\left(F_{+}^{I}(t)\right)^{2}\right), \quad F_{+}^{I}(t) o\left(\frac{1}{x}\right)=o\left(\left(F_{+}^{I}(t)\right)^{2}\right) \quad \text { при } t \rightarrow \infty .
$$

(ii) Пусть выполнены условия раздела II(ii) теоремы 5.2. Здесь в дополнение к $(5.5),(5.7)$ нам понадобится асимптотика $F_{\eta-}(x)$ при $x \rightarrow \infty$. Согласно предположениям имеем $F_{\eta-} \in \mathscr{R}_{\beta_{\eta}}$. Поэтому требуемое утверждение непосредственно вытекает из утверждения (ii) теоремы 1.2 с учетом соотношений, аналогичных (5.8).

(iii) Пусть выполнены условия раздела II(iii) теоремы 5.2. Тогда вновь утверждение теоремы 5.2 вытекает непосредственно из раздела (iii) теоремы 1.2 , примененного к случайным величинам $\eta, Y$.

Теорема 5.2 доказана.

3 а м е ч а н и е 5.2. Теорема 5.2 показывает, что в условиях I, II(i) асимптотика второго порядка $\mathbf{P}(\bar{Z} \geqslant x)$ или не зависит от распределения $\tau$ (в условии I), либо зависит от него лишь через коэффициент $a_{\eta}=-a+q \tau$.

3 а м е ч а н и е 5.3. Теорема 5.2 переносит утверждение теоремы 1.2 на обобщенные процессы восстановления. Совершенно аналогично на обобщенные процессы восстановления можно перенести утверждение теоремы 1.1 в случае $\mathbf{E} \xi^{2}<\infty, \mathbf{E} \tau^{2}<\infty$, пользуясь при $q \leqslant 0$ соотношением $\bar{Z}=Y$ и теоремой 1.1 , примененной к случайным величинам $\eta$ и $Y$. При этом окажется, что второй член асимптотического разложения для $\mathbf{P}(\bar{Z} \geqslant x)$ будет содержать по сравнению с (1.3) дополнительное слагаемое $-q F_{+}(x) \mathbf{E} \tau($ см. (5.7)).

Теоремы 1.1, 1.2, 5.2 позволяют получать асимптотику второго порядка для стационарных распределений времени ожидания и длины очереди в теории очередей, для вероятности разорения в теории страхования, в том числе в задачах о страховании с кредитованием (ср. с [7]). 


\section{СПИСОК ЛИТЕРАТУРЫ}

1. Veraverbeke $N$. Asymptotic behaviour of Wiener-Hopf factors of a random walk. Stochastic Process. Appl., 1977, v. 5, p. 27-37.

2. Боровков A.A. О субэкспоненциальных распределениях и асимптотике распределения максимума последовательных сумм. - Сиб. матем. журн., 2002, т. 43, № 6 , с. $1235-1264$.

3. Боровков A. A., Боровков K. А. Асимптотический анализ случайных блужданий. Т. 1: Медленно убывающие распределения скачков. М.: Физматлит, 2008, 650 с.

4. Феллер B. Введение в теорию вероятностей и ее приложения, т. 2. М.: Мир, 1984, $752 \mathrm{c}$.

5. Боровков A. А. Теория вероятностей. М.: Эдиториал УРСС, 2009, 652 с.

6. Bingham N. H., Goldie C. M., Teugels I. L. Regular Variation. Cambridge: Cambridge Univ. Press, 1987, $491 \mathrm{p}$.

7. Borovkov A.A. Insurance with borrowing. The first- and second-order approximations. - Adv. Appl. Probab., 2009, v. 41, № 4, p. 1141-1160.

8. Geluk J. L., Pakes A. G. Second order subexponential distributions. - J. Aust. Math. Soc. Ser. A, 1991, v. 51, № 1, p. 73-87.

Поступила в редакцию

5.II. 2013 